\title{
Low Gestational Age Associated with Abnormal Retinal Vascularization and Increased Blood Pressure in Adult Women
}

\author{
ANNA KISTNER, LENA JACOBSON, STEFAN H. JACOBSON, ELISABETH SVENSSON, AND \\ ANN HELLSTRÖM \\ Department of Nephrology, Karolinska Hospital, Stockholm, Sweden [A.K., S.H.J.]; Institution of Women's \\ and Child's Health, Karolinska Institute, Stockholm, Sweden [A.K.]; Department of Ophthalmology, \\ Huddinge Hospital, Huddinge, Sweden [L.J.]; Department of Statistics, Örebro University, Örebro, \\ Sweden [E.S.], Department of Clinical Neurosciences, Section of Ophthalmology, University of Göteborg, \\ Göteborg, Sweden [A.H.]; International Pediatric Growth Research Centre, Institution of the Health of \\ Women and Children, The Queen Silvia Children's Hospital, Göteborg, Sweden [A.H.]
}

\begin{abstract}
The objective was to investigate any possible relationship between functional and structural vascular changes in women with low gestational age and/or low birth weight by analyzing the retinal vascular pattern in women with thoroughly documented blood pressure. Retinal vessel morphology was evaluated by digital image analysis of ocular fundus photographs in 47 subjects, aged 23-30 y. The women were allocated into three groups: 1) those born preterm and appropriate for gestational age (AGA), with a median gestational age at birth of $30 \mathrm{wk}$ and a median birth weight of $1250 \mathrm{~g}(n=14) ; 2)$ those born small for gestational age (SGA) but full term (median $40 \mathrm{wk}$ ), with a median birth weight of $2130 \mathrm{~g}(n=17)$, and 3) those born full term, AGA, and with a median birth weight of $3640 \mathrm{~g}(n=16)$. Women born preterm had significantly higher length index for arterioles compared with the other two groups (median 1.11 and 1.08 , respectively, $p=0.005$ ). In addition, the preterm-born women had significantly fewer number of vascular branching
\end{abstract}

\section{ABSTRACT}

points compared with the controls (median 27 and 30, respectively, $p=0.03$ ). The abnormal retinal vascularization observed in ex-preterm women together with an increased casual blood pressure observed in these subjects suggests that being born preterm does have effects on the vascular system that persist into adult life. In addition, it demonstrates that preterm birth seems to affect the vascular system both functionally and structurally, which, in adulthood, could result in a lower threshold for the development of vascular disease. (Pediatr Res 51: 675-680, 2002)
Abbreviations
ROP, retinopathy of prematurity
SGA, small for gestational age
AGA, appropriate for gestational age
GA, gestational age

Preterm birth carries a greatly increased risk of morbidity, manifested, for example, by brain injury and bronchopulmonary dysplasia $(1,2)$. In addition, preterm infants are at risk of developing $\operatorname{ROP}(3,4)$ and cerebral visual impairment (5). During the last decade, several studies have reported that low birth weight is associated with raised blood pressure (6), increased mortality as a result of coronary heart disease (7), and metabolic changes (8). It has been shown that undernutri-

Received June 25, 2001; accepted January 29, 2002.

Correspondence: Ann Hellström, M.D., Ph.D., Section of Pediatric Ophthalmology, The Queen Silvia Children's Hospital, Sahlgrenska University Hospital, S-416 85 Göteborg, Sweden; e-mail: ann.hellstrom@medfak.gu.se

Supported by grants from the Göteborg Medical Society, the FrimurareBarnhusdirektionen, the Swedish Society for Medical Research, and the Swedish Medical Research Council (\#10863 and \#13515).

DOI: 10.1023/01.PDR.0000017482.64723.C4 tion before birth program persisting changes in a range of metabolic and physiologic variables (9). In some of these studies, no relationship was seen between raised blood pressure and GA $(6,10-12)$, although the highest rates of deaths from coronary heart disease occurred in those born preterm $(13,14)$.

Increased blood pressure has been found in ex-preterm adult women (15) compared with subjects born full term, although there was a discrepancy between an increased casual blood pressure and a normal 24-h ambulatory blood pressure.

As preterm birth is associated with adverse effects on the vascular system, manifested as periventricular leukomalacia (16), ROP (17), and an increased frequency of hemangioma (18), there could, in addition, be an early defect in the morphologic development of the vascular system.

The aim of the present study was to investigate the influence of GA and/or birth weight on the design and architecture of the 
arterioles, as these vessels are of particular interest for later development of cardiovascular disease. The retinal vasculature was chosen as the target of the study as, unlike other parts of the vascular system, it allows detailed, noninvasive morphometrical evaluation.

\section{MATERIAL AND METHODS}

Forty-seven women, with a mean age of $26 \pm 2$ y (range 23-30 y) were included in the present study and they were allocated to three groups: 1) preterms, born with a median GA of $30 \mathrm{wk}$ (range 28-32 wk; $n=14$ ), and born AGA, with a median birth weight of $1250 \mathrm{~g}$ (range 950-2040 g); 2) subjects born full term (between gestational wk 38 and 42), with a median birth weight of $2130 \mathrm{~g}$ (range 1610-2660 g; $n=17$ ), and born SGA; and 3) the comparison group, born full term with appropriate birth weight [median $3640 \mathrm{~g}$ (range 3120$4220 \mathrm{~g})]$ for GA $(n=16)$, Table 1 . None of the participants suffered from chronic diseases or were taking medications that might affect blood pressure or the vascular structure.

Anthropometric data. The first day of the last menstrual period before pregnancy and birth weight were obtained from medical records.

GA was estimated according to the first day of the last menstrual period, in cases where the mother was certain of this date. In the remaining five cases, an evaluation of the maturity stage of each baby was performed by a neonatologist at birth.

Birth size was estimated according to the reference by Niklasson et al. (19). Individuals with a birth weight below 2 SD were considered SGA.

Between the years 1970 and 1973, a majority of the preterm individuals, born in Stockholm, Sweden, were treated at the St. Goran Children's Hospital. Nineteen girls were born before 32 gestational weeks, were traceable, and had survived to adult age. Of these 19, 11 women accepted to participate in the present study. In addition, during the years 1973 to 1974,11 women were born in the Stockholm area, with a GA $<30 \mathrm{wk}$, and had survived to adult age according to the National Medical Birth Register. Nine of them could be traced and contacted, and four elected to participate in the study, although one of them was later excluded due to Down syndrome. Thus, 14 individuals born preterm were included. During the time of birth of these children, there was no screening for ROP, however, as found from retrospective file review, only one girl was treated for ROP among the included women.
During 1966-1967, 54 women agreed to take part in a study because they had given birth to a low-weight baby at Sabbatsbergs Hospital, Stockholm. Thirty-two women in the study had female babies, and the protocols of 19 women were traceable. From the neonatal records, 10 of these individuals fulfilled the criteria for the study and were contacted, 6 of these individuals agreed to participate. From the National Medical Birth Register, a total of 20 females, born between the years 1973-1974 in the Stockholm area, were randomly selected and contacted by letters. Eleven agreed to participate in the study. Thus, 17 SGA subjects were included.

Eleven age-matched women born full term with appropriate birth weight in the Stockholm area were randomly selected from the National Medical Birth Register, of these three were born on the same day and in the same hospital as subjects in the other two groups. Furthermore, three were healthy volunteers and two came from neonatal records taken on the same day and in the same hospital as subjects in the other two groups. Thus, 16 individuals were included as a comparison group.

Present clinical history was evaluated and a clinical investigation was performed by a doctor (A.K.) at the outpatient clinic. The Ethics Committee at Karolinska Hospital, Stockholm, approved the study, and the participants included gave their informed consent.

Digital image analysis. Ocular fundus photographs were taken after cycloplegia and analyzed quantitatively, using a specifically designed digital image analysis system (20).

Only correctly focused photographs with the optic disc centered were accepted for analysis. One of the preterm-born women had had proliferate ROP, and had an abnormal ocular fundus and was not included for analysis. Thus 13 preterm women, 17 women born SGA, and 16 controls were included for analysis. Central retinal vascularization was analyzed with respect to the length of arterioles, and venules, and number of vascular branching points (20). Measurements were made, without knowledge of identity and diagnosis of study subjects, by tracing each vessel (path length) from its origin on the optic disc to a reference circle with a radius of $3.0 \mathrm{~mm}$ from the geometric center of the optic disc (Fig. 1). The length of arterioles and venules was expressed as a length index, i.e. the path length of the vessel divided by the linear distance from the vessel origin to the reference circle. Vessels were also traced from their branching point to the reference circle, and the total

Table 1. Weight, length, GA, and ponderal index at birth, and blood pressure (BP) control at follow-up

\begin{tabular}{|c|c|c|c|c|}
\hline & Preterm Group $(n=13)$ & SGA Group $(n=17)$ & Comparison Group $(n=16)$ & $p$ Value* \\
\hline Weight $(g)$ & $1250(950-2040)$ & $2130(1610-2660)$ & $3640(3120-4220)$ & \\
\hline Weight SDS & $-1.91(-2.9,-0.04)$ & $-3.44(-4.43,-2.53)$ & $0.06(-1.50,1.78)$ & \\
\hline Length (cm) & $40(36-43.5)$ & $46(42-49)$ & $51(48-54)$ & \\
\hline Gestational age (wk) & $30(28-32)$ & $40(37.5-42)$ & $40.25(39-42)$ & \\
\hline Ponderal index $\left(\mathrm{kg} / \mathrm{m}^{3}\right)$ & $20(16-25)$ & $23(19-26)$ & $28(26-34)$ & \\
\hline Systolic BP at first examination (mean $\pm \mathrm{SD}$ ) & $123 \pm 13 \$$ & $111 \pm 10$ & $110 \pm 7$ & $<0.01$ \\
\hline
\end{tabular}

Values given as median (range). SDS, standard deviation scores.

$*$ ANOVA, $\dagger$ Kruskall-Wallis test, $\ddagger p<0.005 v s$ control, $\S p=.07 v s$ control, $\llbracket p=.07 v s$ control. 
number of branching points, i.e. the number of retinal vessels within this area, was calculated.

Blood pressure measurements. Casual blood pressure measurements and ambulatory blood pressure measurements were performed in each participant, and the methods are described elsewhere (15). The results from these investigations demonstrated that the preterms had significantly increased casual systolic blood pressure after $15 \mathrm{~min}$ of rest compared with the comparison group $(p<0.005)$ (Table 1$)$. In addition, the preterms showed a significantly increased number of systolic blood pressure recordings $>130 \mathrm{~mm} \mathrm{Hg}$ during 24-h ambulatory blood pressure $(p<0.05)$ as well as a larger integrated area under the curve $>130 \mathrm{~mm} \mathrm{Hg}$ systolic $(p=0.05)$ (Table 1).

Statistical methods. The ocular fundus variables for each individual were defined as the mean of the two eye measurements. The differences between the median values of the fundus variables of the women born preterm, the women born SGA, and the full-term individuals in the comparison group were evaluated by means of the Wilcoxon-Mann-Whitney $U$ test with correction of the $p$ value for multiple comparisons.

\section{RESULTS}

The women born preterm had significantly longer retinal arterioles (median length index 1.11, range 1.10-1.24) compared with both SGA individuals (median length index 1.08, range 1.05-1.13) and the comparison group (median length index 1.08, range 1.04-1.13), $p=0.005$ (Figs. 2 and 3).

Preterm-born women also had a significantly lower number of vascular branching points (median 27, range 19-36) than the comparison group (median 30, range 25-39), $p=0.03$. (Figs. 2 and 3). Women born SGA showed no significant differences in number of vascular branching points compared with the preterm group (median 29, range 21-38), $p=0.12$.

No difference in length index of the retinal venules between the three groups was found in this study.

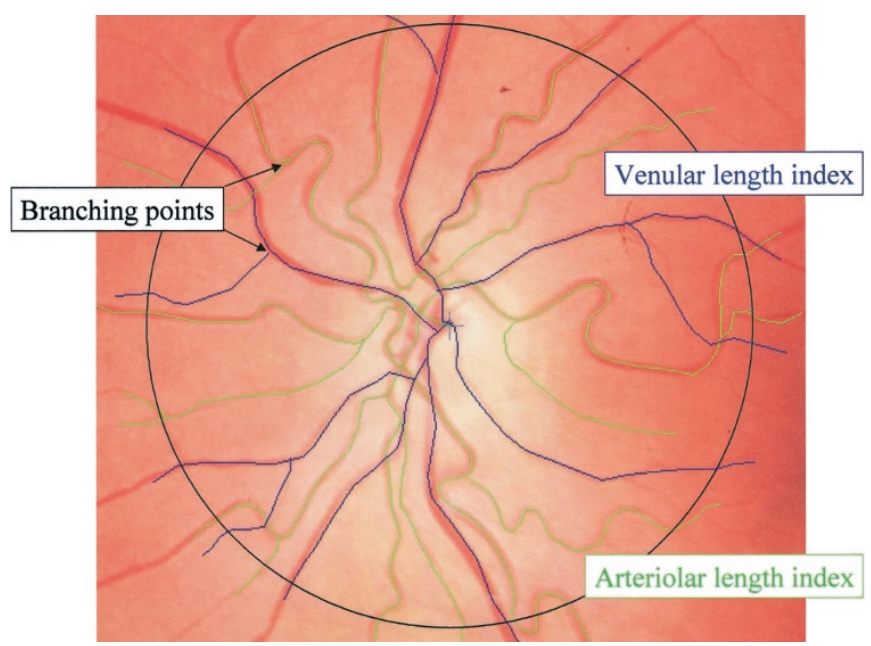

Figure 1. Fundus photograph with digital mapping of fundus variables in an ex-preterm woman with increased length index for arterioles.

\section{DISCUSSION}

Women born preterm have an abnormal vascular pattern. This was demonstrated by an increased length of the retinal arterioles (vessels with a dimension corresponding to the proximal resistance arteries), and by "rarefaction" of the retinal vessels, expressed as a reduced number of vascular branching points (Figs. 2 and 3). Increased retinal vessel tortuosity has been described previously in preterm-born children, independently of ROP but most likely dependent on the fact that the retina is not fully vascularized at birth (21-23), although the implications of this finding were not discussed. In addition, Fryczkowski et al. (24) did not observe any branching arterioles or capillaries using postmortem scanning electron microscopy in a preterm patient. Studies of preterm-born individuals have earlier demonstrated that the vascular abnormalities seemed to be permanent $(21,22,25)$, although the follow-up time has only been to $18 \mathrm{y}$ of age. The findings of abnormal vascular morphology among ex-preterm women at a median age of $25 \mathrm{y}$ clearly suggest that the early perinatal influence on the vascular architecture persists into adulthood.

Preterm birth results in a drastic change in environment. Whereas the full-term baby spends the last months of gestation in the relatively stable intrauterine environment, in which the
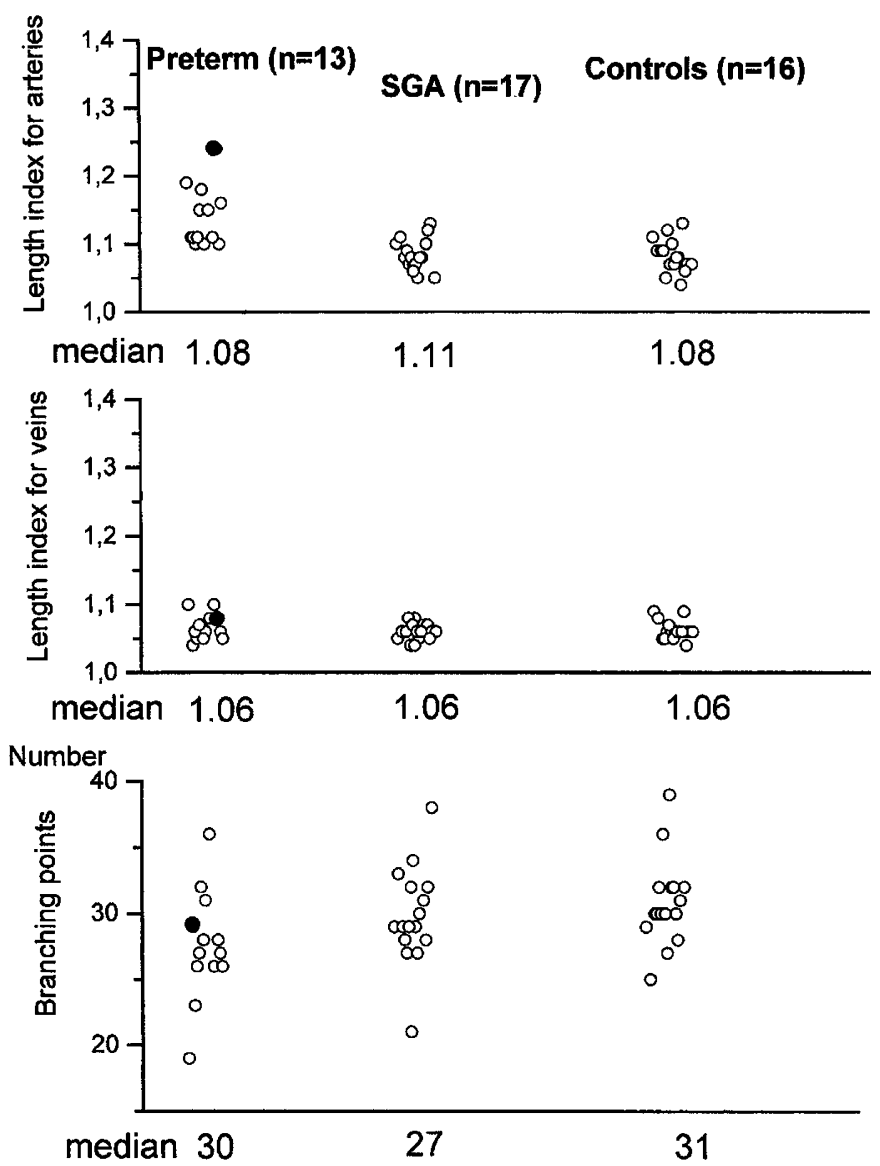

Figure 2. Length index for arterioles and venules and number of retinal vascular branching points in ex-preterm adult women $(n=13)$, women born SGA $(n=17)$, and women born full term with appropriate size $(n=16)$. Solid circles indicate individual values of the preterm-born woman illustrated in Figure 3. 

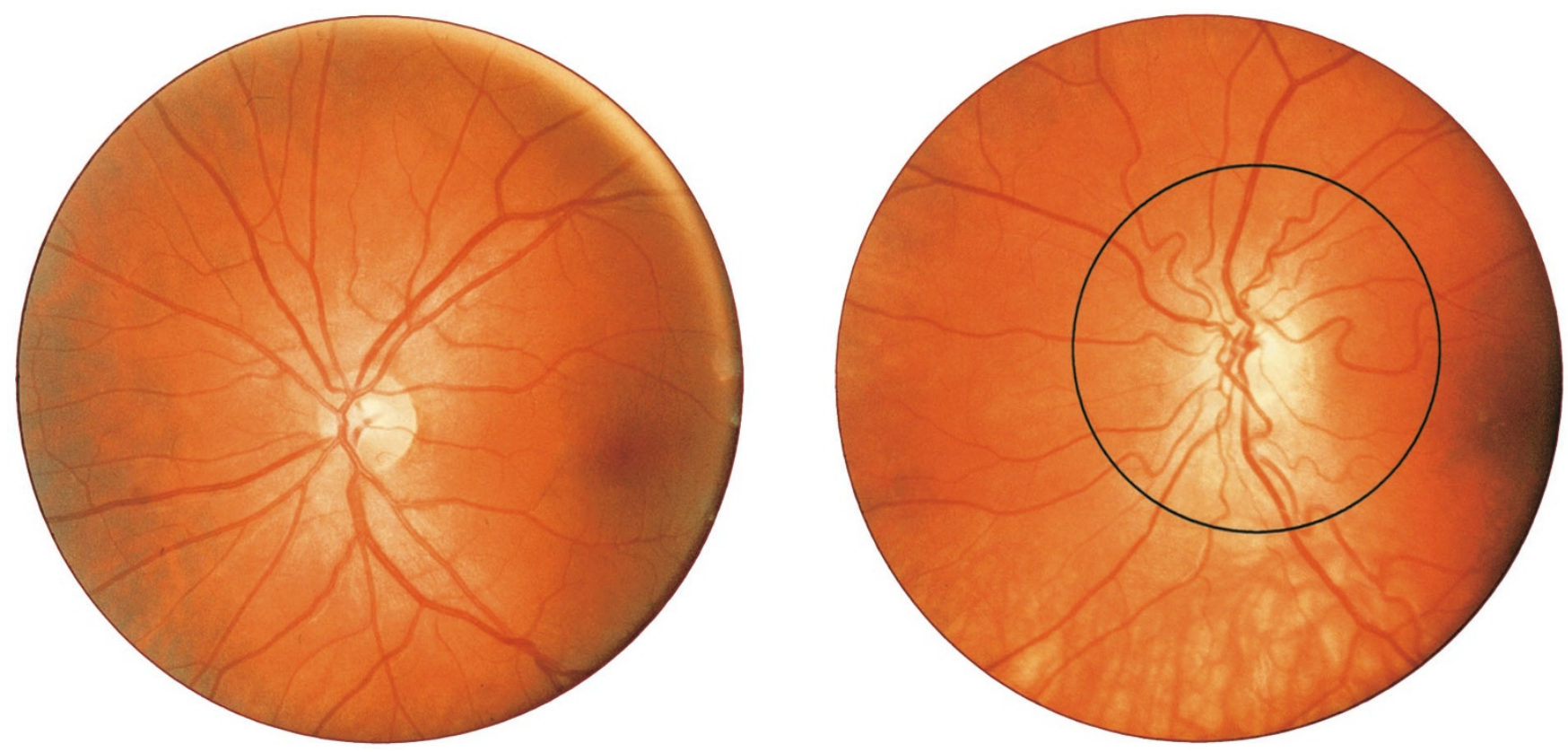

Figure 3. Fundus of 28 -y-old woman born in wk 28 of gestation (right). The central circle depicts the area analyzed by digital mapping. Individual values are depicted as solid circles in Figure 2. The photograph at left is the fundus from a healthy 28 -y-old full-term-born woman with normal vascularization.

growth spurt takes place, the preterm baby spends the corresponding time period in a markedly different environment, with altered functional and metabolic requirements. For example, the preterm baby has to adapt from an intrauterine partial oxygen tension of $40-45 \mathrm{~mm} \mathrm{Hg}$ to an extrauterine tension of $100 \mathrm{~mm} \mathrm{Hg}$, which may considerably alter the development of the vascular system, as suggested by the findings in the present study.

Acute hypoxia, by direct and tissue-mediated effects, relaxes systemic blood vessels, whereas chronic hypoxia increases blood supply capacity by affecting the structural dimensions and architecture of the microvascular network. This seems to be the case particularly during prenatal development, as shown, for example, in chicken embryos by Adair et al. $(26,27)$. Supply arteries become wider, arteriolar and capillary networks become denser, and maximal flow capacity increases dramatically. By analogy, it is likely that chronic hyperoxygenation has marked counterinfluences on vascular design during the important prenatal and perinatal periods when growth tissues are sensitively regulated by a variety of local influences and trophic agents. It is, for example, well known that acute hyperoxygenation powerfully constricts the smooth muscle of systemic blood vessels and, when chronic, it may also affect vascular geometry and architectural development. Preterm infants are, during a decisive period of their early life, chronically exposed to relative hyperoxygenation when compared with the far lower oxygen tension present during fullterm intrauterine life. This may have the opposite structural geometric effects on the microvasculature to those produced by chronic hypoxia, thus explaining the observed rarefaction of retinal artery branching. By further analogy, the retinal arterioles may become structurally narrowed, though this is far more difficult to ascertain with morphometric techniques. A generalized narrowing of smaller retinal arterioles has been found to be strongly related to current blood pressure in both men and women (28). It should be remembered that a reduction of the inner radius of a vessel by only $5 \%$, which is quite difficult to estimate morphometrically, reduces blood flow by as much as $25 \%$ at any given level of vascular smooth muscle tone. This is because flow changes with the fourth power of the radius, according to the law of Poiseuille.

In a preterm child, the peripheral retina is avascular at birth. In this avascular/hypoxic part of the retina, angiogenic factors such as vascular endothelial growth factor are expressed (29). It may be speculated that the increased level of angiogenic growth factors produced by the hypoxic parts of the retina stimulate endothelial cell differentiation in the existing vessels, resulting in vessel elongation. The arteriolar vascular length increase (compared with women born SGA and the comparison group) observed in the present study reduces flow proportionally to the increase in length.

It should be noted that the abnormal vascular pattern is not seen at birth, but is developed around 2-3 mo of age $(21,22$, 30 ), which suggests that the immediate postnatal environment may indeed be responsible for the observed vessel abnormalities.

Could the observed vascular abnormalities in ex-preterm women be a general vascular phenomenon? There is reason to believe that the retinal vascular pattern noted in ex-preterm individuals reflects similar changes in other vascular systems with similar architecture and autoregulation, i.e. the cerebral vessels, the coronary vessels, and the vessels in the kidneys. These are organs in which the vessel design and structure are of particular importance for nutrition and organ function.

During the last decade, several studies have shown that low birth weight is associated with high blood pressure $(6,11)$, ischemic heart disease (7), death from cardiovascular disease $(6,9,14,31,32)$, increased insulin resistance (33), and im- 
paired glucose tolerance (8) later in life. On the basis of these findings, Barker et al. (9) suggested that the disease is "programmed" in utero. This hypothesis proposes that adaptations made by the fetus in response to undernutrition lead to persisting changes in metabolism and organ structure.

As there is a strong relationship between weight at birth and GA, the effect of these variables may be difficult to distinguish from each other. In some of the above-mentioned studies, the association between GA and systolic blood pressure was analyzed, but no relationship was found $(7,10-12)$. In three of these studies, however, the children were aged 4 and $10 \mathrm{y}$, respectively $(6,11,12)$, and the structural vessel changes noted in preterm-born subjects are unlikely to result in clinically detectable increased blood pressure at this early age. In addition, the range of GA in the preterm individuals in these studies was not stated, but it seems likely that the individuals were much less premature than the preterm-born subjects in the present study. It should also be noted that in most of the previous population-based studies, all individuals were included, independent of GA, whereas ex-preterm women and subjects born full term but growth retarded were separated in the present study. Furthermore, the end points of previous studies were outcomes of vascular disease, i.e. blood pressure and cardiovascular disease/death, whereas the present study also focused on morphologic abnormalities of the vascular system.

Hence, our results do not appear to contradict those of previous studies, and they further support previous studies of abnormalities in the retinal vasculature found in preterm-born children aged 4 to 10 y (34). Can the observed abnormal vascular pattern, occurring during the first months of life, and the increased casual blood pressure in ex-preterm women, add to the previously hypothesized "programming" of the fetus as a predisposing factor for vascular disease in adult life? If so, an ex-preterm individual may be fated to have a lower "threshold" for development of increased vascular resistance and restriction of maximal flow capacity than the full-term born, especially when additional age-related morphologic vascular changes or adverse vascular stimuli occur later in life. Stanton et al. (35) found a relationship between increased blood pressure and a decline in arteriolar number in the retinal microvasculature, and a reduced number of perifoveal retinal vessels have also been found in subjects with atherosclerotic disease (36), a relation that increased in patients with both hypertension and arterial disease. In our previous study, the ex-preterm women showed an increased casual blood pressure compared with the comparison group (15), and, assuming that the increased blood pressure precedes the cardiovascular disease, our findings, at least partly, further support this programming theory. However, the mean 24-h blood pressure was not significantly different in the preterm-born subjects, indicating that an increased arousal to psychosocial stress may be involved.

In the women born full term but SGA, we found no significant differences in the retinal vasculature architecture compared with the comparison group. Narrower bifurcation angles have been previously described in elderly men born with low birth weight (37), independent of blood pressure, a morphologic variable that was not analyzed in the present study.
Further studies to clarify the link between the retinal vascular pattern in preterm-born individuals and the development of vascular disease are warranted, particularly as it has been shown previously that ex-preterm individuals have the highest ratio of deaths from cardiovascular disease in adult life (13, 14). It may also be speculated that analysis of the retinal vascular pattern in ex-preterm individuals may identify individuals at risk for more general structural vascular abnormalities.

Acknowledgments. The authors thank Björn Folkow, Professor Emeritus, Department of Physiology, Sahlgrenska University Hospital, and Göran Berglund, Professor, Department of Medicine, University Hospital Malmö, for their most valuable discussions. We also thank Mireille Vanpée, M.D., Ph.D., Institute of Women and Child Health, Karolinska Institute, for help with collecting and distinguishing the preterm material; Bengt Persson, M.D., Ph.D., for providing part of the SGA material; and Petra Otterblad at the National Medical Birth Register, for valuable assistance.

\section{REFERENCES}

1. Hagberg B, Hagberg G, Beckung E, Uvebrant P 2001 Changing panorama of cerebral palsy in Sweden. VIII. Prevalence and origin in the birth year period 1991-94. Acta Paediatr 90:271-277

2. Gorman WA, Fallon M, Kelly M, Clarke T, Griffin E, Matthews T, Murphy J, O'Brien N, Sheridan M 1996 The Dublin outcome for low birth-weight infants. Ir Med J 89:186-187

3. Kocur I, Kuchynka P, Rodny S, Barakova D, Schwartz EC 2001 Causes of severe visual impairment and blindness in children attending schools for the visually handicapped in the Czech Republic. Br J Ophthalmol 85:1149-1152

4. Rowlands E, Ionides AC, Chinn S, Mackinnon H, Davey CC 2001 Reduced incidence of retinopathy of prematurity. Br J Ophthalmol 85:933-935

5. Jacobson L, Lundin S, Flodmark O, Ellström K 1998 Periventricular leukomalacia causes visual impairment in preterm children. Acta Opthalmol Scand 76:593-598

6. Barker DJP, Osmond C, Golding J, Kuh D, Wadsworth MEJ 1989 Growth in utero, blood pressure in childhood and adult life, and mortality from cardiovascular disease. BMJ 298:564-567

7. Barker DJP, Osmond C, Winter PD, Margetts B 1989 Weight in infancy and death from ischaemic heart disease. Lancet 9:577-580

8. Phipps K, Barker DJP, Hales CN, Fall CHD, Osmond C, Clark PMS 1993 Fetal growth and impaired glucose tolerance in men and women. Diabetologia 36:225228

9. Barker DJP, Gluckman PD, Godfrey KM, Harding JE, Owens JA, Robinson JS 1993 Fetal nutrition and cardiovascular disease in adult life. Lancet 341:938-941

10. Barker DJP, Bull AR, Osmond C, Simmonds SJ 1990 Fetal and placental size and risk of hypertension in adult life. BMJ 301:259-262

11. Law CM, Barker DJP, Bull AR, Osmond C 1991 Maternal and fetal influences on blood pressure. Arch Dis Child 61:1291-1295

12. Law CM, de Swiet M, Osmond C, Fayers PM, Barker DJ, Cruddas AM, Fall CH 1993 Initiation of hypertension in utero and its amplification throughout life. BMJ 306:24-27

13. Barker DJP, Osmond C, Simmonds SJ, Wield GA 1993 The relation of small head circumference and thinness at birth to death from cardiovascular disease in adult life. BMJ 306:422-426

14. Martyn CN, Barker DJP, Osmond C 1996 Mothers' pelvic size, fetal growth, and death from stroke and coronary heart disease in men in the UK. Lancet 348:12641268

15. Kistner A, Celsi G, Vanpee M, Jacobson SH 2000 Increased blood pressure but normal renal function in adult women born preterm. Pediatr Nephrol 15:215-220

16. De Reuck J, Chatta A, Richardson E 1972 Pathogenesis and evolution of periventricular leukomalacia in infancy. Arch Neurol 27:229-236

17. 1984 An international classification of retinopathy of prematurity. The Committee for the Classification of Retinopathy of Prematurity. Arch Ophthalmol 102:1130-1134

18. Amir J, Metzker A, Krikler R, Reisner SH 1986 Strawberry hemangioma in preterm infants. Pediatr Dermatol 3:131-132

19. Niklasson A, Ericson A, Fryer JG, Karlberg J, Lawrence C, Karlberg P 1991 An update of the Swedish reference standards for weight, length and head circumference at birth for given gestational age (1977-1981). Acta Paediatr Scand 80:756-762.

20. Hellström A, Svensson E 1998 Optic disc size and retinal vessel characteristics in healthy children. Acta Ophthalmol 76:260-267

21. Mushin AS 1974 Retinopathy of prematurity - a disease of increasing incidence. Transactions of the Ophthalmological Society of the United Kingdom 94:251-257 
22. Fielder AR, Shaw DE, Robinson J, Ng YK 1992 Natural history of retinopathy of prematurity: a prospective study. Eye 6:233-242

23. Hellström A, Hård A-L, Chen Y, Niklasson A, Albertsson-Wikland K 1997 Influence of gestational age, birth size, perinatal morbidity, and postnatal growth. Invest Ophthalmol Vis Sci 38:184-192

24. Fryczkowski AW, Peiffer RL, Merritt JC, Kraybill EN, Eifrig DE 1985 Scanning electron microscopy of the ocular vasculature in retinopathy of prematurity. Arch Ophthalmol 103:224-228

25. Baum JD, Bulpitt CJ 1970 Retinal vasoconstriction in premature infants with increased arterial oxygen tensions. Arch Dis Child 45:350-353

26. Adair TH, Guyton AC, Montani JP, Lindsay HL, Stanek KA 1987 Whole body structural adaption to prolonged hypoxia in chick embryos. Am J Physiol 252:12281234

27. Adair TH, Montani JP, Guyton AC 1988 Effects of intermittent hypoxia on structural vascular adaptation in chick embryos. Am J Physiol 254:1194-1199

28. Sharett AR, Hubbard LD, Cooper LS, Sorlie PD, Brothers RJ, Nieto FJ, Pinsky JL, Klein R 1999 Retinal arteriolar diameters and elevated blood pressure. The atherosclerosis risk in communities study. Am J Epidemiol 150:263-270

29. Pierce EA, Avery RL, Foley ED, Aiello LP, Smith LE 1995 Vascular endothelial growth factor/vascular permeability factor expression in a mouse model of retinal neovascularization. Proc Natl Acad Sci U S A 92:905-909
30. Bracher D 1982 Changes in peripapillary tortuosity of the central retinal arteries in newborns. A phenomenon whose underlying mechanisms need clarification. Graefes Arch Clin Exp Ophthalmol 218:211-217

31. Frankell S, Elwood P, Sweetnam P, Yarnell J, Smith DG 1996 Birthweight, adult risk factors and incident coronary heart disease: the Caerphilly study. Public Health 110:139-143

32. Stein CE, Fall CH, Kumaran K, Osmond C, Cox V, Barker DJP 1996 Fetal growth and coronary heart disease in South India. Lancet 348:1269-1273

33. Phillips DIW, Barker DJP, Hales CN, Hirst S, Osmond C 1994 Thinness at birth and insulin resistance in adult life. Diabetologia 37:150-154

34. Hellström A, Hård AL, Niklasson A, Svensson E, Jacobsson B 1998 Abnormal retinal vascularisation in preterm children as a general vascular phenomenon. Lancet 352:1827

35. Stanton AV, Mullaney P, Mee F, O'Brian ET, O'Malley K 1995 A method of quantifying retinal microvascular alterations associated with blood pressure and age. J Hypertens 13:41-48

36. Ibrahim Y, Bots M, Mulder P, Grobbee D, Hofman A, de Jong P 1998 Number of perifoveal vessels in aging, hypertension, and atherosclerosis: The Rotterdam Study. Invest Ophthalmol Vis Sci 39:1049-1053

37. Chapman N, Mohamudully A, Cerutti A, Stanton A, Sayer AA, Cooper C, Barker D, Rauf A, Evans J, Wormald R, Sever P, Hughes A, Thom S 1997 Retinal vascular network architecture in low-birth-weight men. J Hypertens 15:1449-1453 